\title{
Evaluation of 18279 Blocks in a Pediatric Hospital
}

\author{
Noemi Vicchio ${ }^{1, *} ;$ Valeria Mossetti ${ }^{1}$; Giorgio Ivani ${ }^{1}$ \\ ${ }^{1}$ Department of Anesthesiology and Intensive Care, Regina Margherita Children's Hospital, Turin, Italy \\ ${ }^{*}$ Corresponding author: Noemi Vicchio, Department of Anesthesiology and Intensive Care, Regina Margherita Children's Hospital, P. O. Box: 10126, Turin, Italy. Tel: +39-113135272, E- \\ mail: noemivicchio@yahoo.it
}

Received: August 18, 2014; Revised: September 15, 2014; Accepted: October 18, 2014

Keywords: Regional Anesthesia; Pediatric Hospital; Blocks, Nerve; Anesthesia and Analgesia

\section{Dear editor,}

As the most active American and European Societies (14), we collect data about pediatric regional anesthesia (PRA) to evaluate the reached degree of safety and efficacy over the years. Each block has been recorded in our database since September 1998. After our Institutional Review Board approval, we made a retrospective analysis of the whole period up to November 2013. We recorded patients' age, number of total blocks, number of central and peripheral blocks, single shot and/or continuous infusions, and perioperative complications. Data are expressed as percentage of overall peripheral and central blocks. Age range was one day to 18 years. A total of 18279 blocks were performed, including 53.4\% central blocks and $46.6 \%$ peripheral blocks. Caudal blocks represented $82.1 \%$ of central blocks, thoracic epidurals represented $3.3 \%$ and lumbar blocks $14.6 \%$. Upper limbs blocks were 29.5\% of peripheral blocks and were mainly axillary blocks (20.7\%); other blocks included interscalenic (2.3\%), supraclavicular (1.0\%), infraclavicular (0.1\%), medial nerve $(0.4 \%)$, radial nerve $(0.8 \%)$, ulnar nerve $(0.3 \%)$, and interdigital nerve (3.9\%). Lower limbs blocks represented 39.4\% and comprised the majority of sciatic blocks with lateral approach (20.0\%), femoral blocks (15.9\%). Trunk blocks comprised $28.5 \%$ and consisted mainly of ilioinguinaliliohypogastric nerve blocks (13.7\%), transversus abdominis plane (TAP) blocks (12.7\%), rectus sheath blocks (0.4\%), and penile blocks (9.0\%). Face blocks for head and neck surgery were $2.6 \%$, mainly including infraorbital nerve, supraorbital nerve or superficial cervical blocks.

We recorded 164 peripheral catheter placements (1.9\% of all the peripheral blocks): 20 infraclavicular, 78 femoral, 58 sciatic catheters, and eight TAP catheters. In the whole period, we had 1688 epidural catheter placements (17.3\% of all the central blocks): 259 thoracic and 1429 lumbar. Looking at the trend of peripheral and central blocks during the 15 years, in the recent years we saw an inversion of the trend (Figure 1) with an increase of peripheral blocks with respect to central blocks, in agreement with the literature $(3,4)$. The probable reason is diffusion, thanks to the ultrasound (US) guidance, of particular blocks such as TAP, supraclavicular, obturator or saphenous blocks, never or rarely performed before. Furthermore, the direct visualization of the needle tip and the local spread under the US guide permitted an increase in the success rate of TAP, rectus sheath and ilioinguinal/iliohypogastric blocks, from 94-95\% before the US introduction to $97-98 \%(5,6)$.

In our audit, the perioperative complications rate has been very low even compared with the Pediatric Regional Anesthesia Network (PRAN) data (4): 12 Dura punctures with two headaches, one Horner's syndrome and two infected catheters. Clearly, a weakness of our retrospective study has been the loss of data about long-term postoperative catheter problems, such as dislodgment, occlusion or kinking, which could increase the global complication rate.

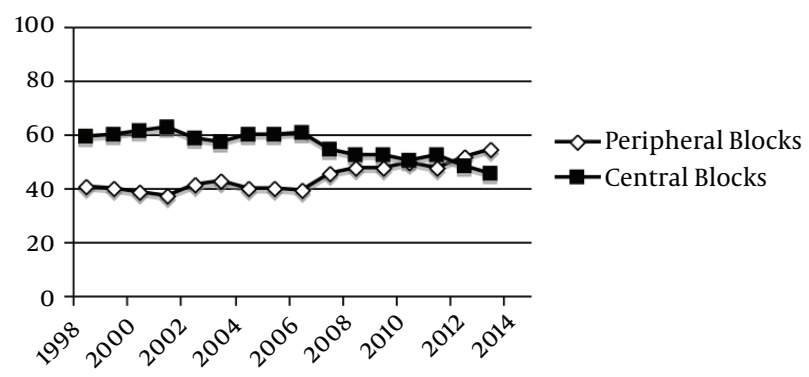

Figure 1. Annual Percentage of Central Blocks Versus Peripheral Blocks During 15 Years of Practice in Regional Anesthesia 
Dural puncture was the most frequent complication (0.1\%) without neurological sequelae. It was associated with headache in one patient, which was solved in less than 24 hours with pain therapy, intravenous infusion and rest. Our Dural puncture rate was lower than the PRAN database (0.9\%) (Eight cases) (4) and similar to the rates of second Association Des Anesthesistes Reanimateurs Pediatriques d'Expression Franeaise (ADARPEF) (0.1\%) (2) as well as the pediatric epidural UK audit (0.07\%) (1).

One case of Horner's syndrome by supraclavicular block was spontaneously solved after 12 hours from the block. In PRAN, Horner's syndrome had a rate of $0.6 \%$, which appeared in patients with thoracic catheters and solved with reduction of the infusion rate (4). Infected catheters were $0.01 \%$ in our report versus $11 \%$ in that of PRAN. Our infections were limited to the insertion site and solved simply by removing the catheters. No caudal catheters were positioned due to higher risk of infections than lumbar catheters, as shown by PRAN (4), and risk of malpositioning and kinking during the cranial advance. No cases of local anesthetic toxicity or respiratory complications were noted after central blocks, probably due to two reasons: the high efficacy of our protocol to prevent spinal injection with spontaneous breathing during slow infusion (7) and repeated aspiration and the exclusion of opioids as adjuvants whenever possible (4).

Our data showed an increase of peripheral blocks than central ones after the US introduction and a high level of efficacy and safety in PRA practice, through following the protocols confirmed by the PRAN data (8). In particular, we recommend the combination of regional and general anesthesia (9) and preservation of spontaneous breathing during regional block procedure. Moreover, in agreement with ADARPEF, when possible, we suggest that peripheral blocks be preferred to central blocks to ensure a greater safety. Prospective studies will be set up in our department as in other pediatric regional anesthesia centers, looking at the comparison of central and peripheral blocks along the years as well as at the complication rate in PRA.

\section{Authors' Contributions}

Study concept and design: Noemi Vicchio, Valeria Mossetti, Giorgio Ivani. Acquisition of data: Noemi Vicchio, Valeria Mossetti. Analysis and interpretation of data: Noemi Vicchio, Valeria Mossetti, Giorgio Ivani. Drafting of the manuscript: Noemi Vicchio, Valeria Mossetti, Giorgio Ivani. Critical revision of the manuscript for important intellectual content: Noemi Vicchio, Valeria Mossetti, Giorgio Ivani. Statistical analysis: Noemi Vicchio, Valeria Mossetti. Administrative, technical, and material support: Noemi Vicchio, Valeria Mossetti. Study supervision: Giorgio Ivani.

\section{References}

1. Llewellyn N, Moriarty A. The national pediatric epidural audit. Paediatr Anaesth. 2007;17(6):520-33.

2. Giaufre E, Dalens B, Gombert A. Epidemiology and morbidity of regional anesthesia in children: a one-year prospective survey of the French-Language Society of Pediatric Anesthesiologists. Anesth Analg. 1996;83(5):904-12.

3. Ecoffey C, Lacroix F, Giaufre E, Orliaguet G, Courreges P, Association des Anesthesistes Reanimateurs Pediatriques d'Expression F. Epidemiology and morbidity of regional anesthesia in children: a follow-up one-year prospective survey of the FrenchLanguage Society of Paediatric Anaesthesiologists (ADARPEF). Paediatr Anaesth. 2010;20(12):1061-9.

4. Polaner DM, Taenzer AH, Walker BJ, Bosenberg A, Krane EJ, Suresh $S$, et al. Pediatric Regional Anesthesia Network (PRAN): a multiinstitutional study of the use and incidence of complications of pediatric regional anesthesia. Anesth Analg. 2012;115(6):1353-64.

5. Willschke H, Marhofer P, Bosenberg A, Johnston S, Wanzel O, Cox SG, et al. Ultrasonography for ilioinguinal/iliohypogastric nerve blocks in children. BrJ Anaesth. 2005;95(2):226-30.

6. Eichenberger U, Greher M, Kirchmair L, Curatolo M, Moriggl B. Ultrasound-guided blocks of the ilioinguinal and iliohypogastric nerve: accuracy of a selective new technique confirmed by anatomical dissection. BrJ Anaesth. 2006;97(2):238-43.

7. Mossetti V, Ivani G. Controversial issues in pediatric regional anesthesia. Paediatr Anaesth. 2012;22(1):109-14

8. Polaner DM, Drescher J. Pediatric regional anesthesia: what is the current safety record? Paediatr Anaesth. 2011;21(7):737-42.

9. Rochette A, Dadure C, Raux O, Troncin R, Mailhee P, Capdevila X. A review of pediatric regional anesthesia practice during a 17-year period in a single institution. Paediatr Anaesth 2007;17(9):874-80. 